\title{
Estandarización, selección individualizada de la técnica quirúrgica, registro de procedimientos y evaluación de la propia evidencia, como componentes esenciales de la práctica quirúrgica moderna
}

\author{
Jorge DAEs, MD, ACC, FACS
}

Palabras clave: cirugía general; historia; procedimientos quirúrgicos; curva de aprendizaje; práctica profesional.

La práctica quirúrgica moderna enfrenta el reto de la actualización en medio del desarrollo vertiginoso de la tecnología y una rápida globalización de la información por medio de internet. El cirujano general se ve tentado a realizar procedimientos mínimamente invasivos sin el entrenamiento adecuado, sin estandarizar su técnica y sin verificar sus resultados.

Los usos sistemáticos de los procesos descritos a continuación ofrecen una salvaguarda a estos problemas y el camino hacia la excelencia quirúrgica.

\section{Estandarización}

La estandarización en cirugía general permanece como una tarea por completar. Hace más de 100 años, en la reunión anual de la American Medical Association de 1914, Dickinson ya se lamentaba de cómo el obrero y el albañil hubieran superado en técnica al cirujano, ya

Fecha de recibido: 9 de octubre de 2017

Fecha de aprobación: 10 de octubre de 2017

Citar como: Daes J. Estandarización, selección individualizada de la técnica quirúrgica, registro de procedimientos y evaluación de la propia evidencia, como componentes esenciales de la práctica quirúrgica moderna. Rev Colomb Cir. 2017;32:247-49. que en sus actividades, los movimientos esenciales, las herramientas y la economía de esfuerzo habían sido estudiadas, estandarizadas y enseñadas, mientras que los cirujanos -científicos de mente abierta- miramos con recelo la aplicación de las mismas medidas ${ }^{1}$. Este lamento viene de un tiempo cuando las técnicas quirúrgicas, los insumos y la tecnología eran limitadas. Los cirujanos, hoy en día, incorporamos nuevas técnicas, insumos y equipos a un ritmo demasiado rápido para permitir una adecuada evaluación y comparación de resultados, así como un control de costos.

A pesar de notables avances en la cirugía de la hernia, la estandarización sigue siendo elusiva ${ }^{2}$. Las redes sociales -una revolución en la enseñanza quirúrgica- con su plétora de publicaciones y comentarios, videos y cirugías en vivo, nos han permitido ser testigos de excepción de este fenómeno, por el cual los cirujanos han acogido una multitud de técnicas y abordajes de mínima invasión para el reparo de la hernia inguinal sin cumplir con las maniobras que la evidencia ha demostrado reducen las complicaciones y el índice de recurrencia.

Un grupo de cirujanos de la International Hernia Collaboration, grupo privado en Facebook con más de 4.000 miembros y una verdadera revolución en la enseñanza de la cirugía de la hernia, conscientes de este 
problema, propusimos una serie de pasos que se deben completar antes de la colocación de la malla, sin importar el tipo de técnica mínimamente invasiva que se emplee, laparoscópica o robótica. Muchas de estas recomendaciones y pasos no son nuevos, han sido enseñados por expertos y por asociaciones científicas por años, y están basados en estudios que demuestran menos recaídas y complicaciones. Este conjunto de recomendaciones las hemos consolidado en el concepto de la vista crítica del orificio miopectíneo ${ }^{3}$.

El concepto de vista crítica fue introducido por Strasberg en 1995 y es estándar de práctica para prevenir las lesiones de la vía biliar durante la colecistectomía laparoscópica. En la cirugía mínimamente invasiva de la hernia inguinal, la vista crítica del orificio miopectíneo se define como la exposición anatómica que debe lograrse siguiendo un conjunto de pasos secuenciales, antes de colocar un material protésico adecuado. Sus objetivos son enseñar y estandarizar el reparo mínimamente invasivo de las hernias inguinales, facilitar la evaluación de videos y cirugías en vivo, reducir las recaídas, prevenir las complicaciones $\mathrm{y}$, finalmente, mejorar el cuidado quirúrgico del paciente.

Hemos observado una referencia constante a los pasos específicos de la vista crítica del orificio miopectíneo en decenas de videos publicados en la International Hernia Collaboration desde la publicación original en Annals of Surgery ${ }^{3}$, con una notable mejoría de la técnica quirúrgica. Un video en el que se describen los pasos de la vista crítica del orificio miopectíneo, se puede observar en:

https://www.facebook.com/jorge.daes/videos/1020367 $6707238837 / ? 1=4600396342676807434$.

La estandarización de las técnicas quirúrgicas ha sido implementada con éxito en otras áreas de la cirugía, como la del cáncer de colon ${ }^{4}$, el programa de Advanced Trauma Life Support ofrecido por el American College of Surgeons y la mejoría sistemática de la morbilidad de la cirugía bariátrica.

Nuestra invocación no es nueva, es una invitación a estandarizar nuestros procedimientos a nivel local, y extenderlos a nivel regional y nacional.

\section{Selección individualizada de la técnica quirúrgica}

La selección de una técnica quirúrgica debe ser adaptada a las características del cirujano, de sus recursos locales, de la enfermedad del paciente y del propio paciente. En el caso de la hernia inguinal, por ejemplo, un cirujano experto en reparo mínimamente invasivo, que trabaja en un sitio con los recursos adecuados, ante una hernia apropiada para el reparo laparoscópico, pero en un paciente con seria disfunción respiratoria, debe elegir una técnica abierta bajo anestesia local, probablemente con la técnica de Lichtenstein. El mensaje es claro: el cirujano debe entrenarse en varios procedimientos para adaptar la técnica al paciente y no lo contrario. En el caso de la hernia inguinal, su repertorio debe incluir una técnica anterior con malla, una posterior con malla, una técnica sin malla y las principales técnicas de mínima invasión.

\section{Registro de procedimientos}

El registro de los procedimientos quirúrgicos en una base de datos se está convirtiendo en una necesidad desde el punto de vista del cirujano, como eventualmente lo será de los sistemas de salud. El registro adecuado de los procedimientos quirúrgicos, como lo han demostrado el registro sueco y el Hernia Med alemán, tiene ventajas como la de permitir una evaluación de los resultados locales y compararlos con los nacionales e internacionales, la mejoría continua de los resultados, la evaluación de la práctica quirúrgica en general y no específicamente en centros de expertos y la detección temprana de efectos secundarios de materiales quirúrgicos; finalmente, sirve de base para estudios con un alto grado de 'evidencia', siempre y cuando el registro sea apropiado. En este momento, la Asociación Colombiana de Cirugía está evaluando la posibilidad de ofrecer a los residentes de cirugía del país un sistema de registro quirúrgico, amigable y seguro, que sirva para iniciar una nueva etapa en la cirugía colombiana.

\section{Evaluación sistemática de resultados}

Finalmente, la evaluación sistemática de los propios resultados, que puede ser complementaria al sistema de registro quirúrgico, es un deber del cirujano. Por un tiempo se estimó que los estudios de metaanálisis responderían nuestros interrogantes sobre las técnicas quirúrgicas óptimas, pero este tipo de análisis está limitado, no solo por 
la falta de rigor en algunos casos, sino por las necesarias barreras que impone la evaluación lineal de problemas biológicos complejos. La evidencia de algunos centros de excelencia no es extrapolable a nuestra realidad local o regional. Es el análisis de nuestros propios resultados, además de la experiencia y el sentido común, lo que nos permite una visión más objetiva de la realidad. La evaluación sistemática de los resultados es un requisito de la difusión de publicaciones de calidad, que constituyen el lenguaje natural entre grupos quirúrgicos.

Estos componentes de la práctica quirúrgica moderna están íntimamente ligados. El registro disciplinado de nuestros procedimientos y su evaluación sistemática, llevan a la identificación de áreas de mejoría y a la estandarización de nuestras técnicas. Es un ciclo que se repite y permite la mejoría continua. Las nuevas técnicas, insumos y tecnologías deben ser evaluados por grupos preparados para dicha investigación y no deben ser acogidos hasta no tener una evidencia de calidad. La cirugía de la hernia está plagada de ejemplos costosos en lo económico y en sufrimiento humano cuando se ha infringido esta regla.

La Asociación Colombiana de Cirugía invita a los residentes y cirujanos en práctica que iniciemos una nueva era que transforme la atención quirúrgica de la nueva Colombia.

\section{Referencias}

1. Dickinson RL. Standardization of surgery: An attack on the problem. JAMA.1914;63:763-5.

2. Daes J. Standardization of hernia surgery. Hernia. 2015;19: 1039-40.

3. Daes J, Felix E. Critical view of the myopectineal orifice. Ann Surg. 20171;266:e1-2.

4. Bertelsen CA, Bols B, Ingeholm P, Jansen JE, Neuenschwander $\mathrm{AU}$, Vilandt J. Can the quality of colonic surgery be improved by standardization of surgical technique with complete mesocolic excision? Colorectal Dis. 2011;13:1123-9.

Correspondencia: Jorge Daes, MD, ACC, FACS

Correo electrónico: jorgedaez@gmail.com

Barranquilla 